\title{
Regression Based Prediction of Minimental State Examination Score Using the Tablet Based Trail Making Test
}

\section{Kouki Kubo}

Hibino Hospital

Seiji Hama ( $\nabla$ shama@hiroshima-u.ac.jp )

Hibino Hospital

\section{Akira Furui}

Hiroshima University

\section{Tomohiko Mizuguchi}

Maxell, Ltd.

\section{Akiko Yanagawa \\ Hibino Hospital \\ Akihiko Kandori \\ Hitachi (Japan) \\ Hiroto Sakai}

Hiroshima University

\section{Yutaro Morisako \\ Hibino Hospital \\ Yuki Orino \\ Hibino Hospital \\ Maho Hamai \\ Hibino Hospital \\ Kasumi Fujita \\ Hibino Hospital \\ Shigeto Yamawaki \\ Hiroshima University \\ Toshio Tsuji \\ Hiroshima University}

\section{Research Article}

Keywords: TMT, PLS, MMSE 
Posted Date: November 25th, 2020

DOI: https://doi.org/10.21203/rs.3.rs-110893/v1

License: (c) (i) This work is licensed under a Creative Commons Attribution 4.0 International License. Read Full License 


\title{
Regression based prediction of minimental state examination score using the tablet based trail making test
}

\author{
Kouki Kubo ${ }^{1}$, Seiji Hama ${ }^{1,2^{*}}$, Akira Furui ${ }^{3}$, Tomohiko Mizuguchi ${ }^{4}$, Akiko Yanagawa ${ }^{1,2}$, Akihiko Kandori ${ }^{5}$, \\ Hiroto Sakai $^{3}$, Yutaro Morisako ${ }^{1}$, Yuki Orino ${ }^{1}$, Maho Hamai ${ }^{1}$, Kasumi Fujita ${ }^{1}$, Shigeto Yamawaki ${ }^{6}$ and Toshio \\ Tsuji $^{*}$
}

\author{
Affiliations: \\ ${ }^{1}$ Department of Rehabilitation, Hibino Hospital, Hiroshima 731-3164, Japan \\ ${ }^{2}$ Department of Neurosurgery, Graduate School of Biomedical and Health Science, Hiroshima University, Hiroshima \\ 734-8551, Japan \\ ${ }^{3}$ Graduate School of Advanced Science and Engineering, Hiroshima University, Higashi-Hiroshima 739-8527, Japan \\ ${ }^{4}$ Optronics Division, Healthcare Systems Business Promotion Project, Maxell, Ltd., Tokyo, Japan \\ ${ }^{5}$ Center for Exploratory Research, Research and Development Group, Hitachi. Ltd., Tokyo, Japan \\ ${ }^{6}$ Center for Brain, Mind and KANSEI Sciences Research, Hiroshima University, Hiroshima 734-8551, Japan
}

\section{Corresponding author: Toshio Tsuji, Seiji Hama}

\section{Seiji Hama}

Department of rehabilitation, Hibino Hospital, Tomo-higashi 7-9-2, Asaminami-ku, Hiroshima 731-3164, Japan Tel: +81-848-2357 Fax: +81-848-

e-mail: shama@hiroshima-u.ac.jp

\section{Toshio Tsuji}

Graduate School of Advanced Science and Engineering, Hiroshima University, Kagamiyama 1-4-1, HigashiHiroshima 739-8527, Japan

Tel: +81-424-7677 Fax: +81-424-2387

e-mail: tsuji@bsys.hiroshima-u.ac.jp

\begin{abstract}
Trail making test (TMT) is one of the most extensively used neuropsychological tests. In this study, we examined the equivalence between the iPad version of TMT part A (iTMT-A) and the paper version of TMT part A (pTMT-A), and predicted the cognitive function with various data extracted from repeated TMT-A. Forty-two patients who performed five repeated TMT-A $\left(1^{\text {st }}-3^{\text {rd: }}\right.$ iTMT-A, $4^{\text {th }}:$ pTMT-A, $5^{\text {th }}$ : inverse version of iTMT-A) and Mini-Mental State Examination (MMSE) were included. The Kruskal-Wallis one-way analysis of variance revealed no statistical differences between the completion times of iTMT-A and pTMT-A. Factors contributing to the MMSE prediction were selected by stepwise multiple regression analysis and Bland-Altman plots. Then, the prediction abilities of the three models-multiple linear, partial least squares (PLS), and neural network regression-were compared. When using the completion time, the linear regression model with the $1^{\text {st }}-5^{\text {th }}$ results exhibited the highest prediction ability. However, when the move time and dwell time were used, the multiple linear and PLS regression models using the $1^{\text {st }}$ and $2^{\text {nd }}$ iTMT-A data exhibited the highest prediction ability. Compared with pTMT-A, iTMT-A extracted a large amount of data with fewer repetitions, and the prediction accuracy of cognitive function was improved.
\end{abstract}

\section{Introduction}

The trail making test (TMT) is one of the most extensively used tests in neuropsychology ${ }^{1,2}$. This test consists of 
two parts: TMT part A (TMT-A) and part B (TMT-B). TMT-A is a task to connect the circled numbers printed on the paper with a line in ascending order from 1 to 25 using a pen or pencil. TMT-B is a task of connecting circled numbers and alphabets printed on paper alternately with lines in ascending order (i.e. 1-A-2-B- ...). TMT is usually evaluated by the completion time, and TMT-B takes longer to complete than TMT- $\mathrm{A}^{2}$. The completion times on the TMT are thought to reflect the visuomotor tracking, divided attention, speed of processing, mental flexibility, and executive function of patients ${ }^{2,3,4}$. However, paper-based TMT (pTMT) has some disadvantages. First, when the subject makes a mistake (e.g. erroneous connection of lines, the line not reaching the circle, and the pen leaving the paper), the examiner points the mistake out to the subject and then corrects it. However, the time required to correct errors differs with each examiner, thus affecting the TMT results ${ }^{1}$. Second, an error may occur when measuring the time using a stopwatch along with the start command ${ }^{1}$.

Recently, digital versions of TMT using iPads and computers have been developed, and their benefits have been reported $^{1,5,6}$. The digital TMT reduces the influence of the examiner and automatically corrects errors. Moreover, the digital TMT could extract various data except for the completion time, that is, segmental analysis based on the use of the dwell or move times and error number. Thus, the digital TMT version would provide new insights that could compensate for the disadvantages of pTMT, which can only extract the completion time. However, these digital versions of the TMT (iPad-based or computer-based TMT) have some differences from the paper version, for example, operation methods (keyboard and mouse for computer, and finger tap or pen for tablet), different familiarity with tablet or computer, and friction on display surface ${ }^{5}$. If the result of the pTMT can be used in the digital version, various data can be extracted by the digital version and analysed by comparing them with the results of the past pTMT. However, the difference in the performance scores across these modes has not been studied in detail, and the reasoning for this is thus unclear ${ }^{5}$.

From these observations, we first examined the equivalence between the digital TMT and pTMT by comparing their completion times. The digital TMT differs from the pTMT because it is operated using a mouse or finger ${ }^{7}$. Thus, in this study, we selected the iPad TMT version (iTMT, constructed with an iPad and Apple Pencil) as the digital TMT version because the operability of iTMT was thought to be closer to that of pTMT. In the comparison with pTMT and iTMT, it is necessary to consider the learning effect of the first attempt on the second attempt. Previous studies have suggested that the learning effect of TMT reached a plateau level within two to three time sessions ${ }^{1}$. Thus, we performed pTMT after performing three sessions of iTMT (after reaching the plateau level of TMT).

The TMT has been used as a cognitive function test and iTMT can extract more factors than pTMT, suggesting that iTMT can estimate cognitive function with higher accuracy than pTMT. Moreover, repeated trials of TMT lead to motor skill learning, which has been known to be one of the important cognitive functions linked with neural multicircuits in many previous studies ${ }^{8}$. Thus, motor skill learning caused by repeated TMT may enable the estimation of non-traditional angles for cognitive function. To test this hypothesis, we performed five repeated TMT tests $\left(1^{\text {st }}\right.$ to $3^{\text {rd }}$ using iTMT, $4^{\text {th }}$ using pTMT, and a $5^{\text {th }}$ test with an alternated inverse version of iTMT, mirrored as the up-down and right-left conversions), and then examined the effect of the various data extracted from these trials on the cognitive function. We herein describe the advantages of iTMT, which are not afforded by conventional pTMT as a cognitive function test.

\section{Methods \\ Subjects}

The ethics committee of Hiroshima University (E-1554-2) and Hibino Hospital approved this prospective study. Written informed consent was obtained from all patients prior to participation. The patients were selected from a consecutive series of individuals who were admitted for rehabilitation to Hibino Hospital for less than 3 months. Exclusion criteria included (1) the history of major psychiatric illness; (2) medical illness, physical disability or speech impediment, which precludes both TMT-A and cognitive function testing. The remaining 42 patients (age: $69.7 \pm 13.5$; male 31 , female 11) were included as subjects in this study (Table 1). This study complied with relevant 
guidelines and regulations in accordance with the Declaration of Helsinki.

\section{Cognitive function test}

We evaluated the cognitive function of patients using the mini-mental state examination (MMSE) that consisted of 11 items with a total score of 30 .

\section{Measurements using pTMT version}

We used the TMT described previously ${ }^{4-11}$; its schema is presented in Figure 1. The pTMT was administered according to the standard protocol after practice using the shortened version of pTMT (search number from 1 to 10). The patient draws a continuous line on the paper as quickly as possible without lifting the pen from the test sheet. If a subject made a mistake (erroneous connecting lines) during the test and did not correct it, the study investigator then immediately corrected them orally. The completion time to connect all 25 items on both sheets was measured, including the extra time required for the administrator to correct for errors.

\section{Measurement using iPad version of TMT}

To study the digital TMT, we used iPad Pro (12.9 in, version 10.2.1, Apple Inc., CA, USA), and lines were drawn using the Apple Pencil. The number displayed on the iTMT screen was the same as that on pTMT. We performed iPad-based TMT (iTMT) after practice using the shortened version of iTMT, similar to pTMT.

In the previous reports on digital TMT, the number of circles was changed after passing through the correct circle, and the parts outside the circumference of a circle were ignored ${ }^{1}$. Moreover, errors classified as passes in incorrect circles resulted in the circles flashing some colours ${ }^{1}$. However, our study compares iTMT and pTMT, wherein the circles would not change their colours, irrespective of whether the circle that passed through was correct or incorrect. If a subject made a mistake (erroneous connection of lines) during the test and did not correct it, the study investigator immediately corrected them orally, similarly to the pTMT case.

As in the alternative version, we created three patterns of the mirror version (right-left, up-down, and both) of the number displayed on the iTMT screen. In this study,
Figure 1

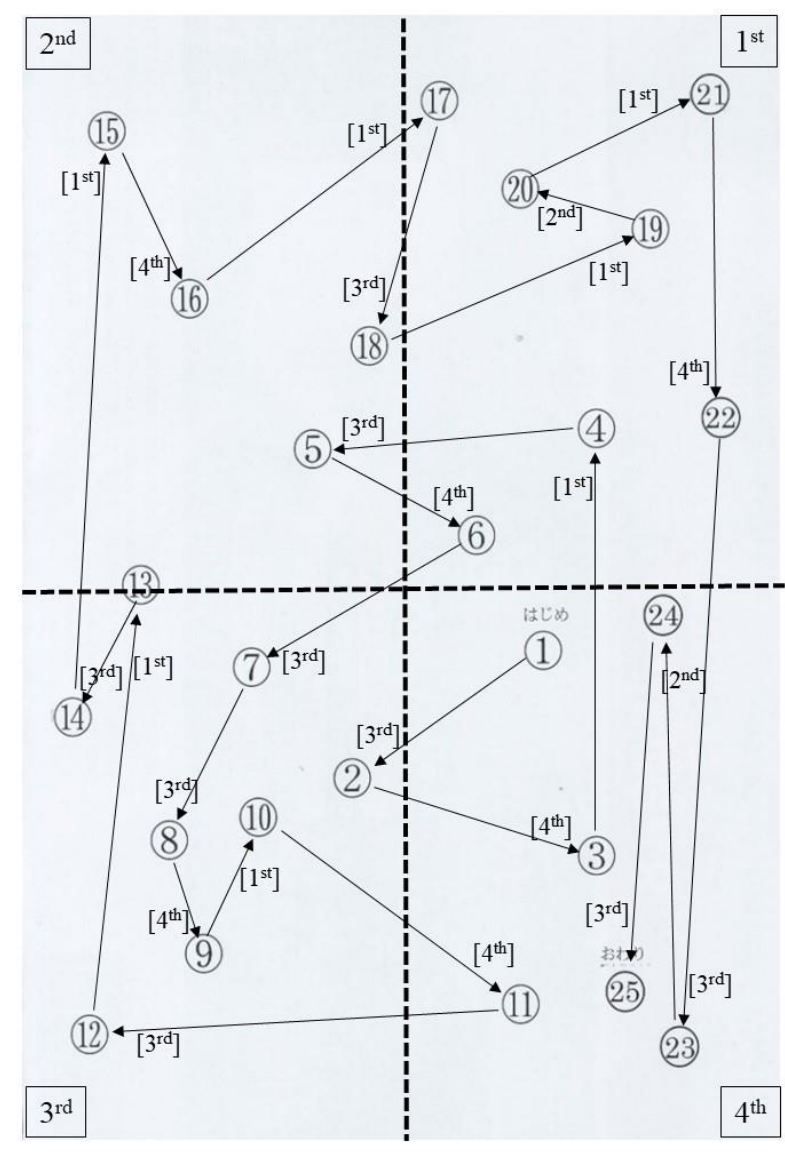
we used the mirror version of both right-left and up-down conversion as the alternative version of the $5^{\text {th }}$ TMT.

To use factors other than the completion time, we measured the dwell time and move time on a segment-by-segment basis and the 'delayed time' (the time from the signal onset to the time the Apple Pencil was placed on the surface of the iPad) ${ }^{1}$. The move time is the time required to move the Apple Pencil. The dwell time is the time required to look for the next target number and was calculated by subtracting the move time from each segment time. However, the subjects constantly moved the Apple Pencil, and it was not possible to clearly distinguish when the pencil was moving and when it was stationary. Therefore, in this study, we set a virtual circle, which was 1.5 times larger than the actual circle (1.5-circle); then, the definition of the dwell time was the time from entering the 1.5-circle to exiting the 1.5circle. When exiting or entering the 1.5 -circle, the dwell time was defined as the time of entering the 1.5 -circle to 
the time of the last exit from the 1.5-circle (without considering going in and out before the final exit of the 1.5circle). The move time was defined as the time from the end of the dwell time to the entry time to the next 1.5 -circle. The quadrant is the angle from the horizontal axis: $0^{\circ}-90^{\circ}$ is the first, $90^{\circ}-180^{\circ}$ is the second, $180^{\circ}-270^{\circ}$ is the third, and $270^{\circ}-360^{\circ}$ is the fourth. The dwell time and move time were grouped into these four quadrants depending on the position of the iPad screen $\left(1^{\text {st }}\right.$ to $4^{\text {th }}$ quadrant of position) and the line drawing direction ( $1^{\text {st }}$ to $4^{\text {th }}$ quadrant of direction) (see Figure 1).

In the preliminary study, we performed an iTMT on healthy volunteers, in which the following problems arose: (1) it was easier to slip on the surface of iTMT than that of pTMT, (2) pass a little outside the circle (within 1.5 circle), and (3) the size of iTMT was smaller than the size of pTMT. To address issue (1), we only used the same version of iPad and Apple Pencil. For issue (2), we classified the pass-through threshold as the circumference of the circle with a radius that was 1.5 times the radius of the original circle. For issue (3), iTMT and pTMT were compared after correcting the result of pTMT in terms of size. Specifically, the length of the line of iTMT was 0.83 times shorter than that of pTMT; thus, the result of pTMT was multiplied by 0.83 to become comparable to the result of iTMT.

\section{Statistical analysis}

Kruskal-Wallis one-way analysis of variance was used to examine the differences in the completion time of the sequential trail tests $\left(1^{\text {st }}\right.$ to $\left.5^{\text {th }}\right)$. Stepwise multiple linear regression analysis based on the Akaike information criterion (AIC) was used to estimate the independent effects of predictor variables on the MMSE score (forward-backward selection method). These predictor variables were as follows: age, sex, laterality of the performed hand, time from the signal onset to the onset of writing with the Apple pen, and $1^{\text {st }}-5^{\text {th }}$ completion time or $1^{\text {st }}-3^{\text {rd }} d w e l l$ and move times; these were divided by quadrants of position and direction, as described in Table 2. This stepwise analysis was of two types: (1) the analysis performed using the completion time of the $1^{\text {st }}$ time, $1^{\text {st }}$ and $2^{\text {nd }}$ time, $1^{\text {st }}$ to $3^{\text {rd }}$ time, $1^{\text {st }}$ to $4^{\text {th }}$ time, or $1^{\text {st }}$ to $5^{\text {th }}$ time TMT-A, and (2) the analysis performed using the move time and dwell time divided into four quadrants of direction or position of the $1^{\text {st }}$ time, $1^{\text {st }}$ and $2^{\text {nd }}$ time, or $1^{\text {st }}$ to $3^{\text {rd }}$ time iTMT-A. Multicollinearity was assessed by examining the variance inflation factor (VIF). A value of 10 was considered to be sufficiently large to indicate the problem of multicollinearity ${ }^{12,13}$. The predicted MMSE score using the developed equations was compared with the measured MMSE score $(n=42)$ using a Bland-Altman plot and correlation coefficients.

Subsequently, the MMSE value was predicted by a multiple regression model, partial least squares (PLS) regression model, and neural network model using the selected predictor variables. The prediction performance of each model was evaluated through a leave-one-subject-out cross-validation (i.e. $k$-fold cross-validation with $k=42$ ).

A PLS analysis was performed to identify combinations of the predictors that had a large covariance with the response values, thus solving the multi-collinearity problem caused by many variables. We retained the smallest number of latent factors for which the predicted residual error sum-of-squares was minimum. The importance of each predictor in the construction of the PLS was assessed based on the importance of the variable in projection scores of old individuals.

The neural network regression model was of a multilayer perceptron type, the number of hidden layers was set to 1 , and the number of units in the hidden layer was set to $\mathrm{D}+10$ (D represents the number of input dimensions). In addition, we introduced $\mathrm{L} 2$ regularisation (i.e. weight decay) to suppress overfitting.

To compare the performance of each model, we calculated three metrics between the actual MMSE and predicted MMSE obtained from all folds: the squared correlation coefficient $\left(r^{2}\right)$, root-mean-square error (RMSE), and mean absolute error (MAE). The values were considered significant at $\mathrm{p}<0.05$. All data were statistically analysed using $\mathrm{R}$ (version 3.6.2).

\section{Results}

\section{Baseline data of subjects}


Forty-nine patients were included in this study, as seven patients could not undergo TMT-A and 18 patients could not complete the TMT-B test. Fourteen patients had impaired cognitive function (MMSE score $<24$ ), four patients could not complete the TMT-A test, and 12 patients could not complete the TMT-B test. The number of cognitiveimpaired patients who could perform TMT-B was only two. Thus, we examined the data of TMT-A and not TMT-B. The number of subjects who performed TMT-A analysed, herein (as described below), was 42. Their baseline characteristics are listed in Table 1.

\begin{tabular}{|l|c|}
\hline Table 1. Basic Charateristics of subjects who could performed TMT-A \\
\hline & All (n=42) \\
\hline Age & $69.7 \pm 13.5$ \\
\hline Sex (male: female) & $31 ; 11$ \\
\hline $\begin{array}{l}\text { type of disease } \\
\text { infarction }\end{array}$ & 23 \\
\hline hemorrhage & 7 \\
\hline subarachnoid hemorrhage & 1 \\
\hline Head injury & 5 \\
\hline others & 6 \\
\hline MMSE score & $26.1 \pm 3.7$ \\
\hline completion time (sec) & $62.9 \pm 35.5$ \\
\hline 1st TMT & $57.4 \pm 26.0$ \\
\hline 2nd TMT & $51.3 \pm 22.3$ \\
\hline 3rd TMT & $50.4 \pm 27.1$ \\
\hline 4th TMT & $56.6 \pm 39.5$ \\
\hline 5th TMT & \\
\hline $\begin{array}{l}\text { TMT: Trail making test, TMT-A: Trail making test part-A. MMSE: Mini- } \\
\text { mental state examination }\end{array}$ & \\
\hline
\end{tabular}

\section{Comparison between iTMT-A and pTMT-A from five repeated TMT-A trials}

The completion times of five repeated TMT-A trials were compared using multiple comparison analysis (KruskalWallis followed by the post-hoc test) (see Table 1, Figure 2). The completion time was shortened from the $1^{\text {st }}$ TMT-

Figure 2

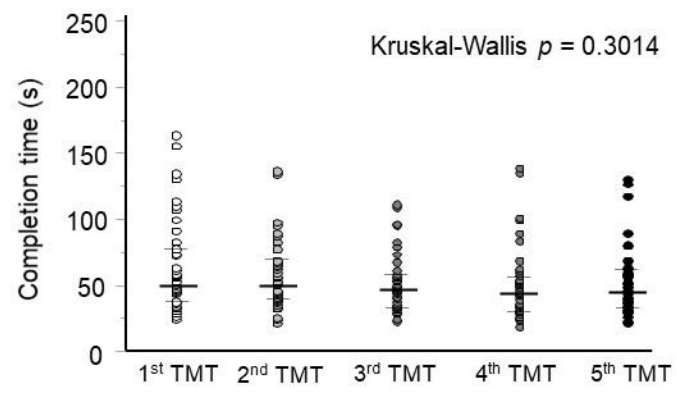

A to the $3^{\text {rd }}$ TMT-A; however, no statistically significant difference was observed.

We performed the $5^{\text {th }}$ iTMT-A (i.e. the up-down and left-right mirror reversed version of the $1^{\text {st }}$ to $4^{\text {th }}$ TMT-A) and demonstrated that its completion time had no remarkable differences. This suggests that there was minimal subject memorisation of the position of the numeral, if any.

\section{Variable selection using stepwise multiple regression analysis}

To determine which of the variables extracted from repeated TMT-A measurements contributed significantly to the prediction of MMSE score, we used stepwise multiple regression analysis (Table 2). The variance inflation factor was $<10.0$, indicating low collinearity. 
Table 2. Stepwise multiple regression analysis predicting MMSE score.

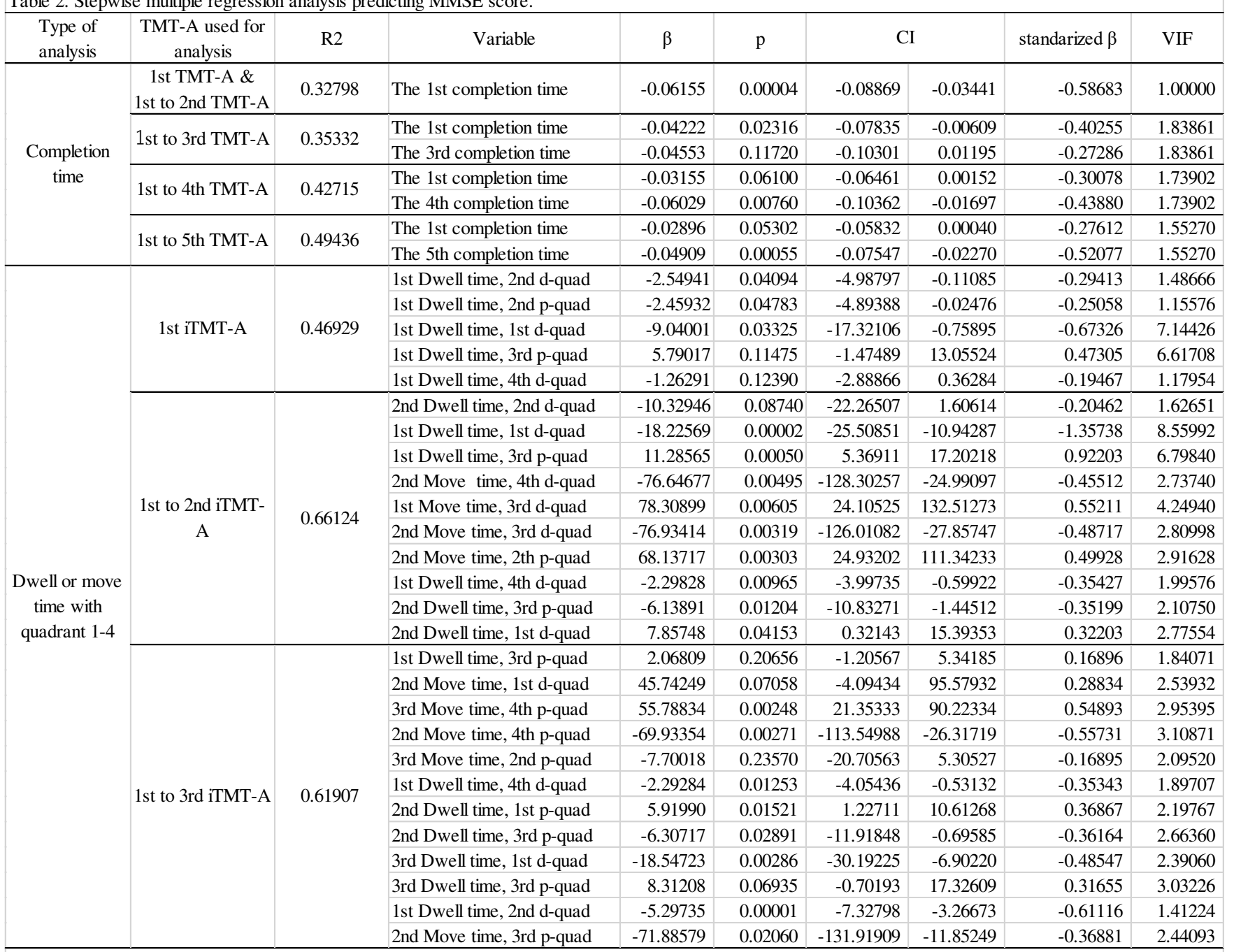

The stepwise analysis was performed using two types of analysis: the analysis performed using the completion time and the analysis performed using the move-time and dwell time divided into 4 quadrants of direction and position.

Factors to be input in all stepwise analysis: age, sex, laterality of the performed hand the time from starting signal to starting writing with the apple pen.

The results are displayed in order of the importance.

R2: adjusted coefficiencies of determination, $\beta$ : regerssion coefficiencient, CI: standard partial regression coefficiencient, VIF: variance inflation factor. TMT-A: trail making test part-A. d-quad: the quadrant of direction, p-quad: the quadrant of position.

The factors that contributed to the prediction of the MMSE were selected by stepwise multiple regression analysis, and the error characteristics of these models were evaluated for the factors obtained using the Bland-Altman plot. In the repeated measurement of only the completion time, the analysis using the measurement results from the $1^{\text {st }}$ to the $5^{\text {th }}$ TMT-A showed the highest $\mathrm{R}^{2}$ value. Conversely, in the repeated measurement of the dwell time and move time categorised with quadrants, the analysis using the $1^{\text {st }}$ and $2^{\text {nd }}$ measurement results had the highest $\mathrm{R}^{2}$ value. Figure 3 shows the Bland-Altman plot, demonstrating that the prediction accuracy is higher in the analysis using the dwell and move times of the
Figure 3

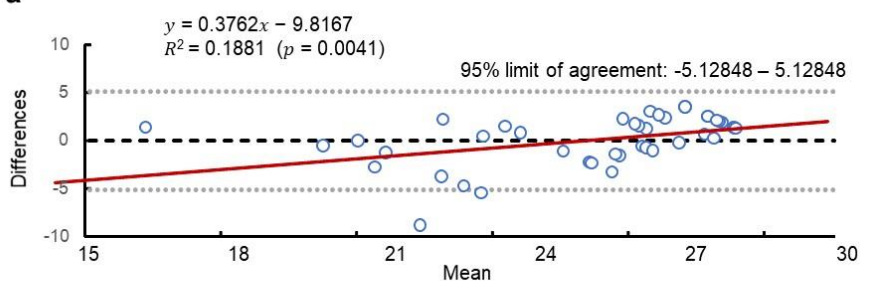

b

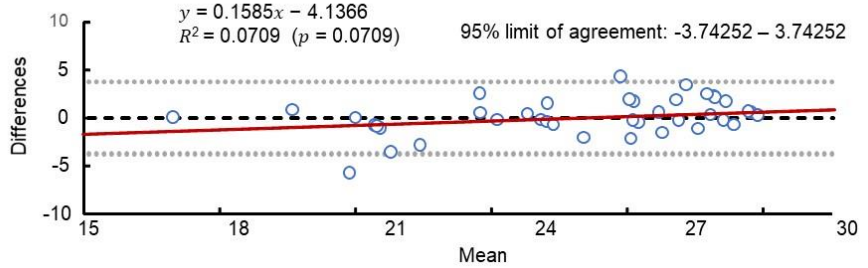


$1^{\text {st }}$ and $2^{\text {nd }}$ iTMT-A (Figure 3-b) than in the analysis that used the completion time of the $1^{\text {st }}$ to the $5^{\text {th }}$ TMT-A (Figure $3-\mathbf{a})$.

\section{Predictions of MMSE score using repeated TMT-A: Comparison of three models}

Table 3 shows the $r^{2}$, RMSE, and MAE values used to compare the three prediction models, and Figure 4 shows the scatter plot of predicted MMSE vs. actual MMSE of the three predicted models.

\begin{tabular}{|c|c|c|c|c|c|}
\hline $\begin{array}{l}\text { Type of } \\
\text { analysis }\end{array}$ & $\begin{array}{l}\text { TMT-A used for } \\
\text { analysis }\end{array}$ & Model & $r^{2}$ & RMSE & MAE \\
\hline \multirow{12}{*}{$\begin{array}{l}\text { Completion } \\
\text { time }\end{array}$} & \multirow{3}{*}{$\begin{array}{c}\text { 1st TMT \& } \\
\text { 1st to 2nd TMT }\end{array}$} & Multiple linear regression & 0.27780 & 3.18411 & 2.60513 \\
\hline & & PLS regression & 0.27780 & 3.18411 & 2.60513 \\
\hline & & Neural network regression & 0.31806 & 3.08132 & 2.51923 \\
\hline & \multirow{3}{*}{ 1st to 3rd TMT } & Multiple linear regression & 0.31042 & 3.11311 & 2.47572 \\
\hline & & PLS regression & 0.32308 & 3.07390 & 2.46550 \\
\hline & & Neural network regression & 0.26360 & 3.25226 & 2.59513 \\
\hline & \multirow{3}{*}{ 1st to 4th TMT } & Multiple linear regression & 0.39159 & 2.91618 & 2.38827 \\
\hline & & PLS regression & 0.39159 & 2.91618 & 2.38827 \\
\hline & & Neural network regression & 0.25569 & 3.22824 & 2.46435 \\
\hline & \multirow{3}{*}{ 1st to 5th TMT } & Multiple linear regression & 0.46827 & 2.73814 & 2.18939 \\
\hline & & PLS regression & 0.48462 & 2.67630 & 2.11043 \\
\hline & & Neural network regression & 0.40081 & 2.88676 & 2.31436 \\
\hline \multirow{9}{*}{$\begin{array}{l}\text { Dwell or move } \\
\text { time with } \\
\text { quadrant } 1-4\end{array}$} & \multirow{3}{*}{ 1st TMT } & Multiple linear regression & 0.36833 & 3.20405 & 2.35474 \\
\hline & & PLS regression & 0.42892 & 2.82061 & 2.20032 \\
\hline & & Neural network regression & 0.32772 & 3.13304 & 2.28775 \\
\hline & \multirow{3}{*}{ 1st to 2 nd TMT } & Multiple linear regression & 0.59642 & 2.41278 & 1.86700 \\
\hline & & PLS regression & 0.59642 & 2.41278 & 1.86700 \\
\hline & & Neural network regression & 0.38009 & 2.99475 & 2.28738 \\
\hline & \multirow{3}{*}{ 1st to 3rd TMT } & Multiple linear regression & 0.41319 & 4.50177 & 2.34457 \\
\hline & & PLS regression & 0.50549 & 2.75969 & 2.11656 \\
\hline & & Neural network regression & 0.51880 & 2.64823 & 2.02905 \\
\hline
\end{tabular}

The MMSE score was predicted by a multiple regression model, PLS regression model, and neural network model using variables selected by Stepwise anlaysis. These 3 models were performed using two types of analysis: The analysis performed using the completion time and the analysis performed using the move-time and dwell time divided into 4 quadrants of direction and position.

$\mathrm{r}^{2}$ : the squared correlation coefficient, RMSE: root mean square error, MAE: mean absolute error. TMTA: trail making test part-A.

\section{Figure 4}
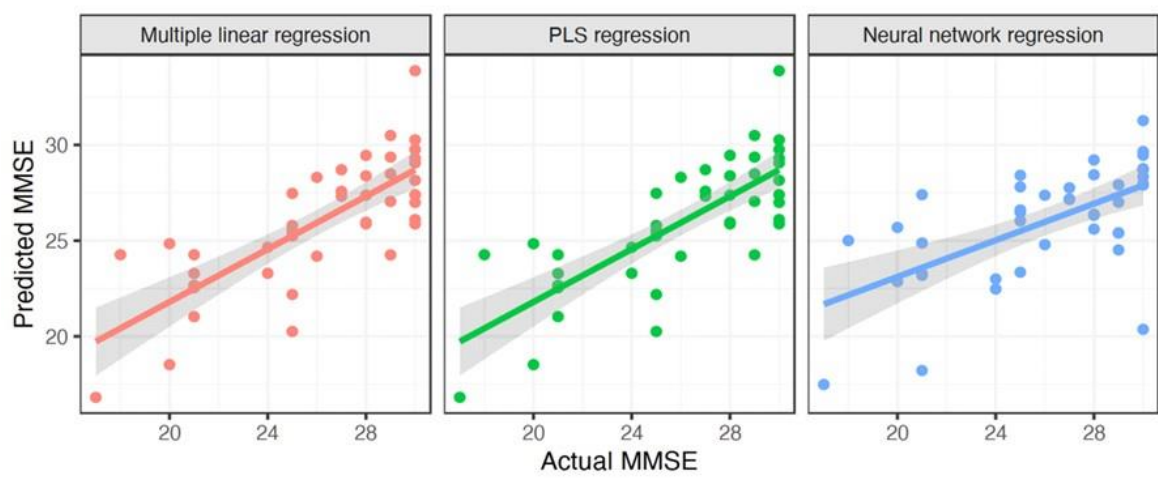

First, when comparing only the completion time, the squared correlation coefficient was low only for the completion time of $1^{\text {st }}$ time TMT-A and for the completion time of the $1^{\text {st }}$ and $2^{\text {nd }}$ times TMT-A. However, it gradually increased after adding the completion time of $3^{\text {rd }}$ time TMT-A in the analysis and became the highest value for the completion time of $1^{\text {st }}$ to $5^{\text {th }}$ times TMT-A (multiple regression model: $r^{2}=0.4683$, PLS regression model: $r^{2}=0.4846$, neural network regression model: $\left.r^{2}=0.4008\right)$. 
Next, we examined the completion time by dividing it into dwell time, move time, and quadrants. The squared correlation coefficients of the multiple linear and PLS regression models were the highest at the $1^{\text {st }}$ to $2^{\text {nd }}$ times (but decreased at the $1^{\text {st }}$ to $3^{\text {rd }}$ times) and exceeded the results of the completion time of the $1^{\text {st }}$ to $5^{\text {th }}$ times of the measurements. Because the value increases as the number of times increases, there is a problem of multi-collinearity after the $3^{\text {rd }}$ time measurement when the data extracted from iTMT-A is divided into many factors. Conversely, the squared correlation coefficient of the neural network regression model was highest at the $1^{\text {st }}$ to $3^{\text {rd }}$ times and exceeded the results of the completion times of the $1^{\text {st }}$ to the $5^{\text {th }}$ measurements.

Figure 4 compares the actual and predicted MMSE values of three predicted models based on a scatter plot. The models with the smallest error (low RMSE and MAE) and the highest estimation ability (high $r^{2}$ value) are the multiple linear and PLS regression models using the dwell time and move time divided into four quadrants of two repeated iTMT-A.

\section{Discussion}

We used iTMT-A to compare its completion time with pTMT-A. According to previous reports, the digital TMT-A and pTMT-A are different in terms of several aspects such as their operation methods, arrangement of numbers, habituation of operations, and slippage of digital surfaces ${ }^{5,7}$. In this study, the same versions of iPad and Apple Pencil were used with the same number arrangement as in pTMT-A. By correcting the distance, we could not identify any significant differences between the completion times of iTMT-A and pTMT-A.

An analysis of the predictive ability of the cognitive function indicated that the predictive ability increased as the number of repetitions of TMT-A increased (highest at the $5^{\text {th }}$ repetition) if only the simple completion time was used. This repetitive effect is believed to be associated with motor skill learning. The motor skill learning was acquired to balance the speed and accuracy of gaze-anchoring and reaching movement. Further, it was regulated based on a series of the visual-perceptual-attention-motor programme-upper-limb-movement control function with the involvement of a large area of the brain ${ }^{14}$. In previous reports, the cognitive functions were mainly correlated with TMT-B and not TMT-A ${ }^{15}$, while TMT-B could not be easily performed for many cognitive-impaired patients. However, our present results suggest that with repeated measurement of TMT, it may be possible to predict cognitive functions sufficiently with part A instead of part B.

A more detailed analysis of iTMT-A was performed using the dwell time and move time after implementing the four quadrants of direction and position, showing that the results of the two measurements exceeded the predictive ability with a simple completion time. The move time, in this study, was believed to reflect the line-drawing motion. TMTA is a task used to identify two targets on a paper or screen and connect these two targets with a line using a pen (or Apple Pencil). In the process, the reach and line-drawing motion of the upper limb and multi-cognitive functions (i.e. visual search, visual-motor tracking, scanning, divided attention, and cognitive flexibility) to co-ordinate them were required $^{1}$. The control of upper-limb movements consisted of the complexity of the shoulder, elbow, and wrist movements that provided considerable flexibility ${ }^{16}$. To control this flexibility, multi-joint movement, a miscellaneous cognitive brain function was required.

The dwell time, in this study, is believed to reflect the time search for the next target and the planning to the upper limb movement. In reaching motion to a target, the gaze was directed towards a visual target until hand movement was completed (gaze-anchoring behaviour) ${ }^{17}$. From the theory of sensorimotor transformation to reach a target, the target location was perceived and stored in an eye-centred frame of reference, transformed to head-centred, shouldercentred, and hand-centred frames to plan multi-joint upper limb movement ${ }^{18}$. To perform TMT-A, two different functions, namely, target recognition and upper limb movement control, need to be coordinated with each other.

The quadrant of direction may be assumed to be slightly involved in the allocation of attention, which is known to be biased towards the objects in the space around the hand (especially the dominant side for right-handed individuals), which biases the allocation of visual-spatial resources towards the contralateral left portion of the space ${ }^{18}$. Moreover, the quadrant of position may be slightly related to the advantages of ipsilateral reaching movement (i.e. shorter 
movement time and higher peak velocity, compared with those of contralateral reaching $)^{18}$.

Given that the move time, dwell time, and quadrant of direction and position involve different brain functions, it was considered that the cognitive function can be easily estimated with a small number of repetitions (two times, in this study) by extracting and analysing many factors from one test. In daily clinical practice, it is difficult to analyse the dwell time and move time separately for each quadrant in the paper version; however, it is easier using the iPad version. The use of iTMT-A instead of pTMT-A is considered to be greatly advantageous.

Most of the remaining factors in this study were dependent on the dwell time and direction quadrant. Given that the conventional paper version focusses on the position quadrant, the conventional pTMT-A exhibits a large variation in the ratio of the four quadrants of direction. Based on this study, it is suggested that the number arrangement should consider the direction quadrant in estimating the cognitive function. In the future, by creating a TMT-A model specialised for iPads, it is expected that it will be easier to estimate the brain function.

\section{Study Limitations}

In this study, we examined the cognitive function using only the MMSE. Many factors obtained by iTMT-A were considered to be involved in many different cognitive functions. To improve the prediction ability of cognitive function, it is necessary to compare it with other brain function test outcomes in the future.

The detection capability of the neural network regression was low. The remaining factors of the stepwise regression model were selected within the framework of the multiple regression model. Owing to its high expressiveness, it is easy to overfit data sets with large variability and small sample size. Patients with cognitive decline often could not complete the TMT-A test, and fewer patients with low MMSE scores were analysed in this study. Further studies are required to increase the number of patients with a wide range of cognitive functions by adding patients with cognitive decline, such as patients who could only complete TMT-A.

In this study, the dwell time and movement time were calculated based on a distance of 1.5-times the target circle radius. In the future, it will be necessary to introduce a method that can accurately evaluate the time to move the pen and the time to search for targets, so that a more detailed analysis can be performed.

\section{Conclusions}

In this study, there were no remarkable differences between the results of iTMT-A and pTMT-A, suggesting that the obtained results can be evaluated in the same way.

Regarding the completion time of the TMT-A, the result obtained by repeating the measurement five times estimated the MMSE score to some degree. Furthermore, when the iTMT-A results were interpreted separately in terms of the dwell time, move time, and quadrants of direction and position, the prediction accuracy of MMSE was the highest of the two measurements.

iTMT-A remains capable of extracting more factors than those used in this study. By comparing and examining the relationships between many factors that can be extracted from iTMT-A and many other brain functions, it will be possible to analyse a wide range of brain functions with iTMT-A in the future. Nevertheless, prior to accomplishing this, it is necessary to compare more factors extracted from iTMT-A with various other cognitive functions.

\section{References}

1. Woods, D. L., Wyma, J. M., Herron, T. J., Yund, E. W. The Effects of Aging, Malingering, and raumatic Brain Injury on Computerized Trail-Making Test Performance. PLOS ONE 10: e0124345. https://doi:10.1371/journal.pone.0124345 (2015).

2. Karimpoor, M., et al. Tablet-Based Functional MRI of the Trail Making Test: Effect of Tablet Interaction Mode. Front. Hum. Neurosci. 11:496. https://doi: 10.3389/fnhum.2017.00496 (2017). 
3. K,Fällman, K., Lundgren, L. L., Wressle, E., Marcusson, J., Classon, E. Normative data for the oldest old: Trail Making Test A, Symbol Digit Modalities Test, Victoria Stroop Test and Parallel Serial Mental Operations. Neuropsychol Dev Cogn B Aging Neuropsychol Cogn. 27:567-580. https://doi: 10.1080/13825585.2019.1648747 (2020).

4. Reitan, R. M. The Relation of the Trail Making Test to Organic Brain Damage. $J$ Consult Psychol. 19:393-4. https://doi: 10.1037/h0044509 (1955).

5. Rodriguez, F. S., et al. Relevance of the assessment mode in the digital assessment of processing speed. J. Clin. Exp. Neuropsychol. 41:730-739. https://doi: 10.1080/13803395.2019.1616079 (2019).

6. Bracken, M. R., Mazur-Mosiewicz, A., Glazek, K. Trail Making Test: Comparison of paper-andpencil and electronic versions. Appl. Neuropsychol. Adult. 26:522-532. https://doi: 10.1080/23279095.2018.1460371. (2019).

7. Sacco, G. et al. Comparison between a Paper-Pencil Version and Computerized Version for the Realization of a Neuropsychological Test: The Example of the Trail Making Test. J Alzheimers Dis. 68:1657-1666. https://doi: 10.3233/JAD-180396 (2019).

8. Dayan, E. and Cohen, L. G. Neuroplasticity Subserving Motor Skill Learning. Neuron. 72:443454. https://doi: 10.1016/j.neuron.2011.10.008 (2011).

9. Abe, M., et al. [Normative data on tests for frontal lobe functions: Trail Making Test, Verbal fluency, Wisconsin Card Sorting Test (Keio version)]. No To Shinkei. 56:567-574. (2004) Japanese.

10. Hirota C., et al. Association between the Trail Making Test and physical performance in elderly Japanese. Geriatr. Gerontol. Int. 10: 40-47. https://doi: 10.1111/j.14470594.2009.00557.x (2010)

11. Armitage, S. G. An Analysis of Certain Psychological Tests Used for the Evaluation of Brain injury. Psychological Monographs,60:1-48 http://doi.org/10.1037/h0093567 (1946).

12. Robert A. Stine R. A. Graphical Interpretation of Variance Inflation Factors. American Statistician 49: 53-56 https://doi.org/10.1080/00031305.1995.10476113 (1995).

13. Zhang, H., Si, Y., Wang, X., Gong, P. Environmental Drivers and Predicted Risk of Bacillary Dysentery in Southwest China. Int. J. Environ. Res. Public Health 14:782. https://doi: 10.3390/ijerph14070782 (2017).

14. Maeda, R. S., Cluff, T., Gribble, P. L., Pruszynski, J. A. Compensating for intersegmental dynamics across the shoulder,elbow, and wrist joints during feedforward and feedback control. J. Neurophysiol. 118:1984-1997. https://doi: 10.1152/jn.00178.2017 (2017).

15. de Assis Faria, C., Alves, H. V. D., Charchat-Fichman, H. The most frequently used tests for assessing executive functions in aging. Dement. Neuropsychol. 9:149-155. https://doi: 10.1590/198057642015DN92000009 (2015).

16. N. Dounskaia, N., Ketcham, C. J., Stelmach, G. E. Commonalities and differences in control of various drawing movements. Exp. Brain Res. 146:11-25. https://doi: 10.1007/s00221-0021144-3 (2002).

17. Rinaldi, L., Di Luca, S., Toneatto, C., Girelli, L. The effects of hemispheric dominance, literacy acquisition, and handedness on the development of visuospatial attention: A study in preschoolersand second graders. J. Exp. Child Psychol. 195:104830. https://doi: 10.1016/j.jecp.2020.104830 (2020).

18. Rand, M. K., and Rentsch, S. Eye-hand coordination during visuomotor adaptation: effects of hemispace and joint coordination. Exp. Brain Res. 235:3645-366. https://doi: 10.1007/s00221017-5088-z (2017). 


\section{Acknowledgements}

We wish to express our gratitude to members of the Kaifukuki Rehabilitation ward and the rehabilitation department and information managers of Hibino Hospital for helping us with the data collection for this study. We also wish to thank Dr. Yasuyuki Kinoshita for his help with the measurement setup in this study. This study was supported in part by a Grant-in-Aid for Early-career Scientists (17K17987).

\section{Author contributions}

Kouki Kubo and Seiji Hama wrote the manuscript; Seiji Hama, Akira Furui, Sakai, and Toshio Tsuji devised the data analysis algorithm; Tomohiko Mizuguchi developed the analytical software (iTMT); Akiko Yanagawa, Yutaro Morisako, Yuki Orino, Maho Hamai, Kasumi Fujita were responsible for data collection and acquisition; Akihiko Kandori shared his expert knowledge in the expert assessment experiment; Seiji Hama and Toshio Tsuji provided their essential ideas for the study. All the authors reviewed the manuscript.

\section{Additional information}

\section{Competing interests}

The authors declare no competing interests.

\section{Data availability}

The datasets generated and/or analysed in this study are available from the corresponding author upon reasonable request.

\section{Figure legends}

\section{Figure 1.}

Trail making test (TMT) part A. Dotted lines indicate the limits of the four quadrants of position. The quadrant number is indicated with a boxed number. The direction of the drawn line between the nodes is indicated with an arrow, and the quadrant number is indicated within parenthesis.

\section{Figure 2.}

Scatter diagrams showing the differences of the completion time in the sequential number of TMT-A $\left(1^{\text {st }}\right.$ to $\left.5^{\text {th }}\right)$. Each circle represents a patient, while the lines indicate the median (bold line) and the quartiles (thin line). The KruskalWallis test of the five repeated TMT-A showed no significant difference $(p=0.3014)$.

\section{Figure 3.}

Bland-Altman plot for the actual mini-mental state examination (MMSE) score and the predicted MMSE score using (a) the compression time of five repeated TMT-A and (b) the move and dwell times divided into four quadrants of duplicated iTMT-A. The black dotted line in boldface and thin black dotted line indicate the mean difference and $95 \%$ limit of agreement, respectively. The red solid line is a regression line between the actual and predicted MMSE score.

\section{Figure 4.}

Scatter plot of predicted MMSE vs. actual MMSE of three prediction models (multiple linear regression, partial least squares (PLS) regression, and neural network regression models). The solid lines represent simple linear regression lines between actual and predicted MMSEs. The shaded area indicates the $95 \%$ confidence interval of each regression line. The prediction performance of each model was calculated by the leave-one-subject-out cross-validation. 
Figures

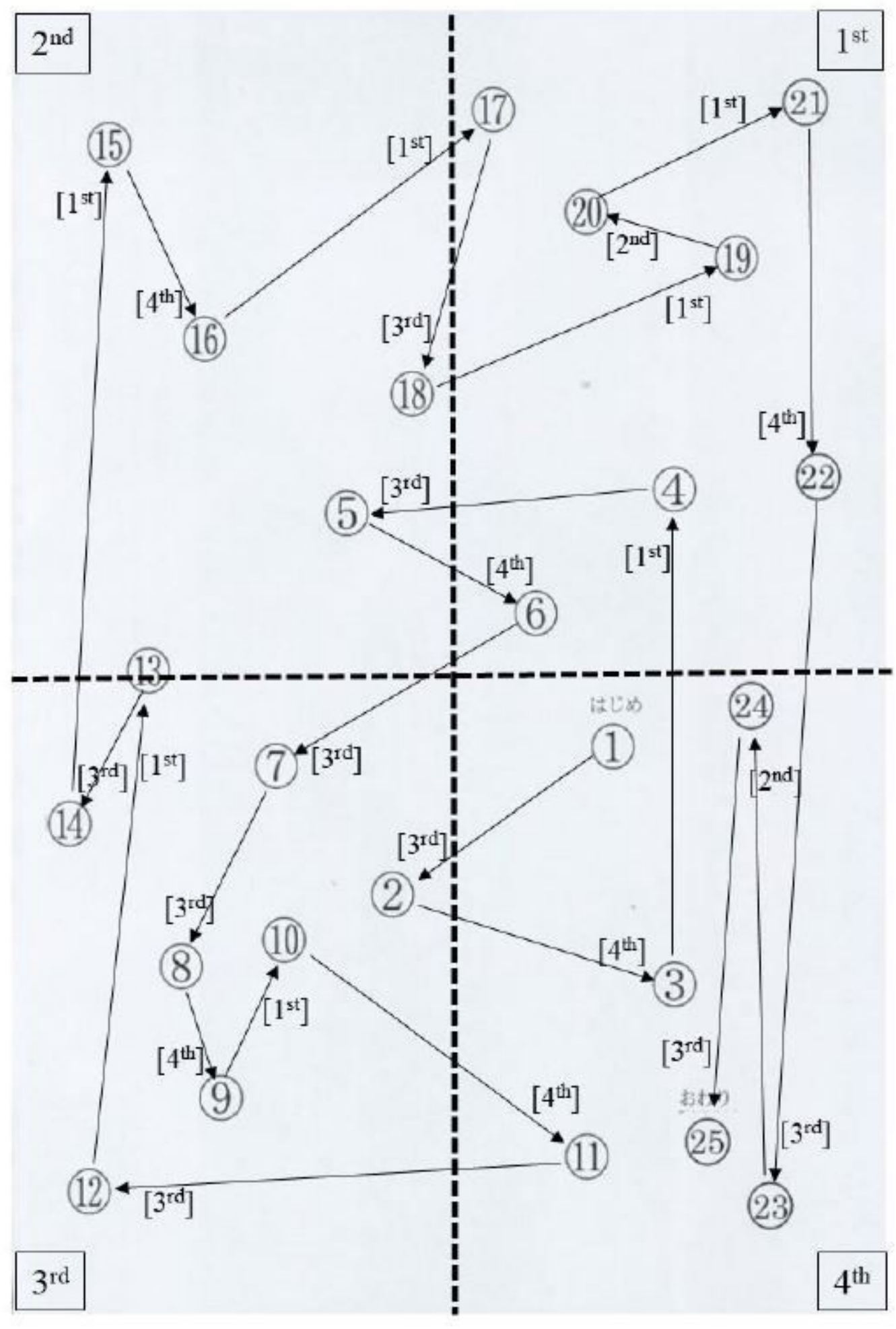

Figure 1

Trail making test (TMT) part A. Dotted lines indicate the limits of the four quadrants of position. The quadrant number is indicated with a boxed number. The direction of the drawn line between the nodes is indicated with an arrow, and the quadrant number is indicated within parenthesis. 


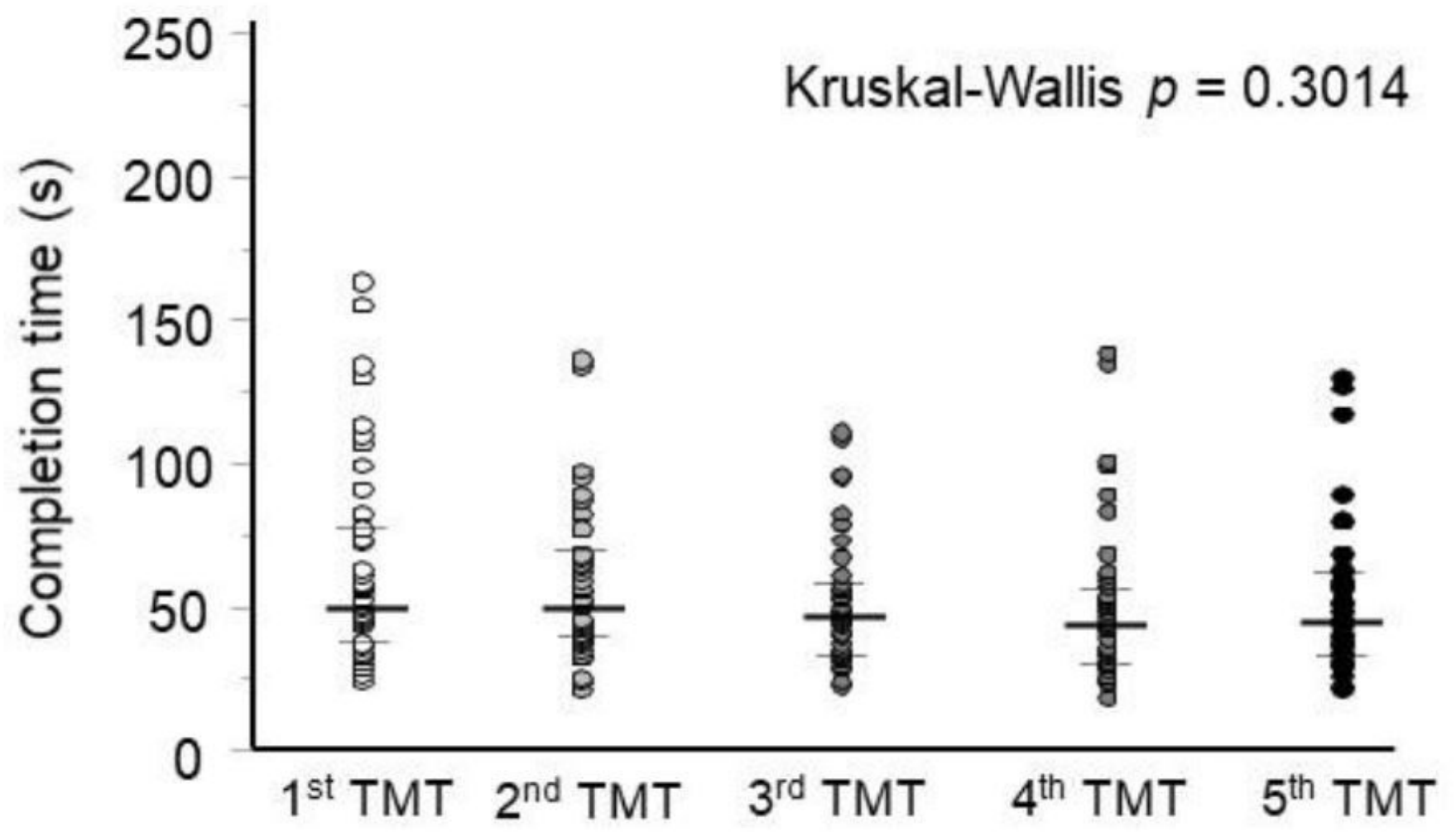

Figure 2

Scatter diagrams showing the differences of the completion time in the sequential number of TMT-A (1st to 5th). Each circle represents a patient, while the lines indicate the median (bold line) and the quartiles (thin line). The Kruskal-Wallis test of the five repeated TMT-A showed no significant difference ( $p=$ 0.3014). 
a

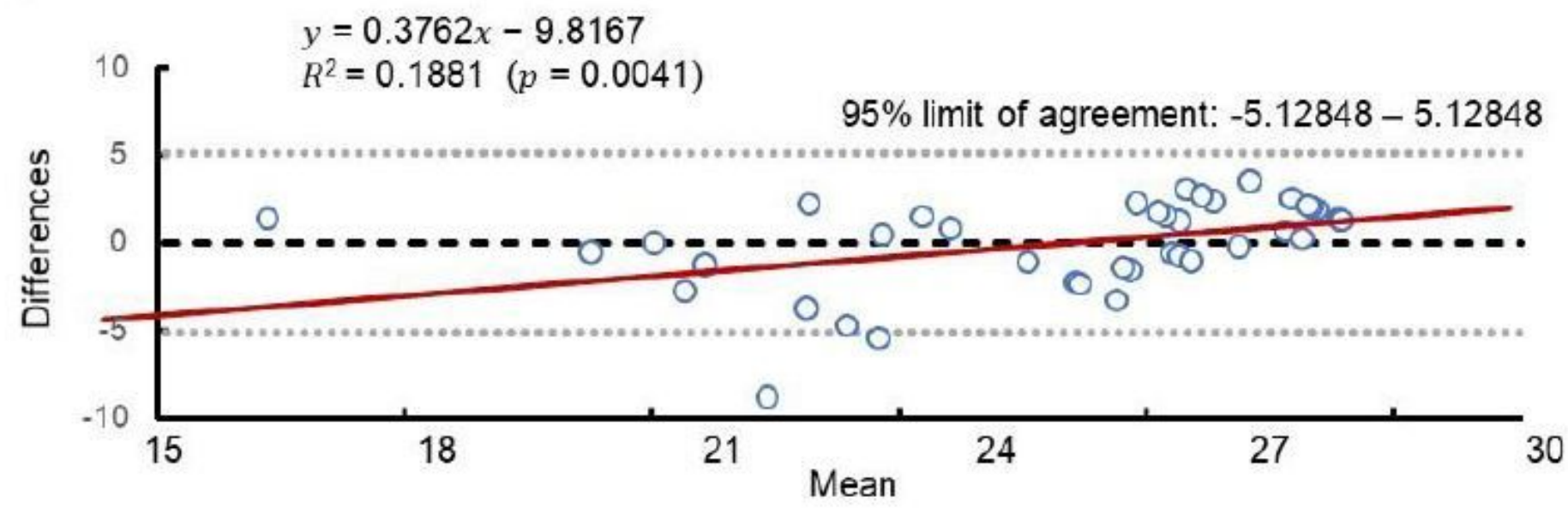

b

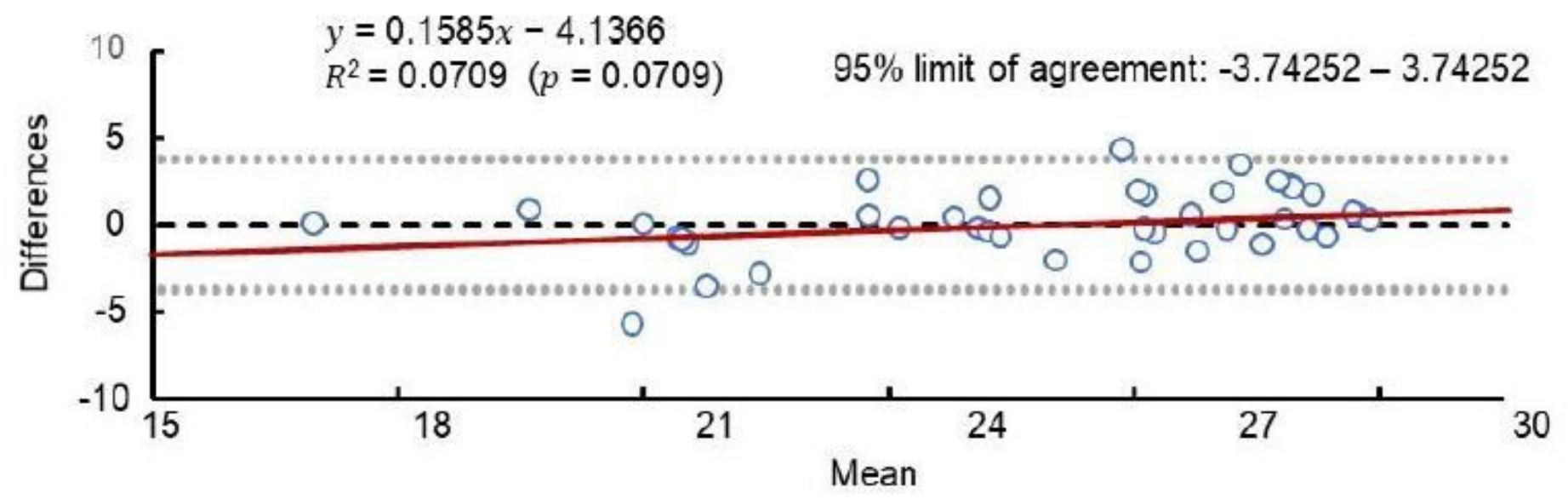

Figure 3

Bland-Altman plot for the actual mini-mental state examination (MMSE) score and the predicted MMSE score using (a) the compression time of five repeated TMT-A and (b) the move and dwell times divided into four quadrants of duplicated iTMT-A. The black dotted line in boldface and thin black dotted line indicate the mean difference and $95 \%$ limit of agreement, respectively. The red solid line is a regression line between the actual and predicted MMSE score. 

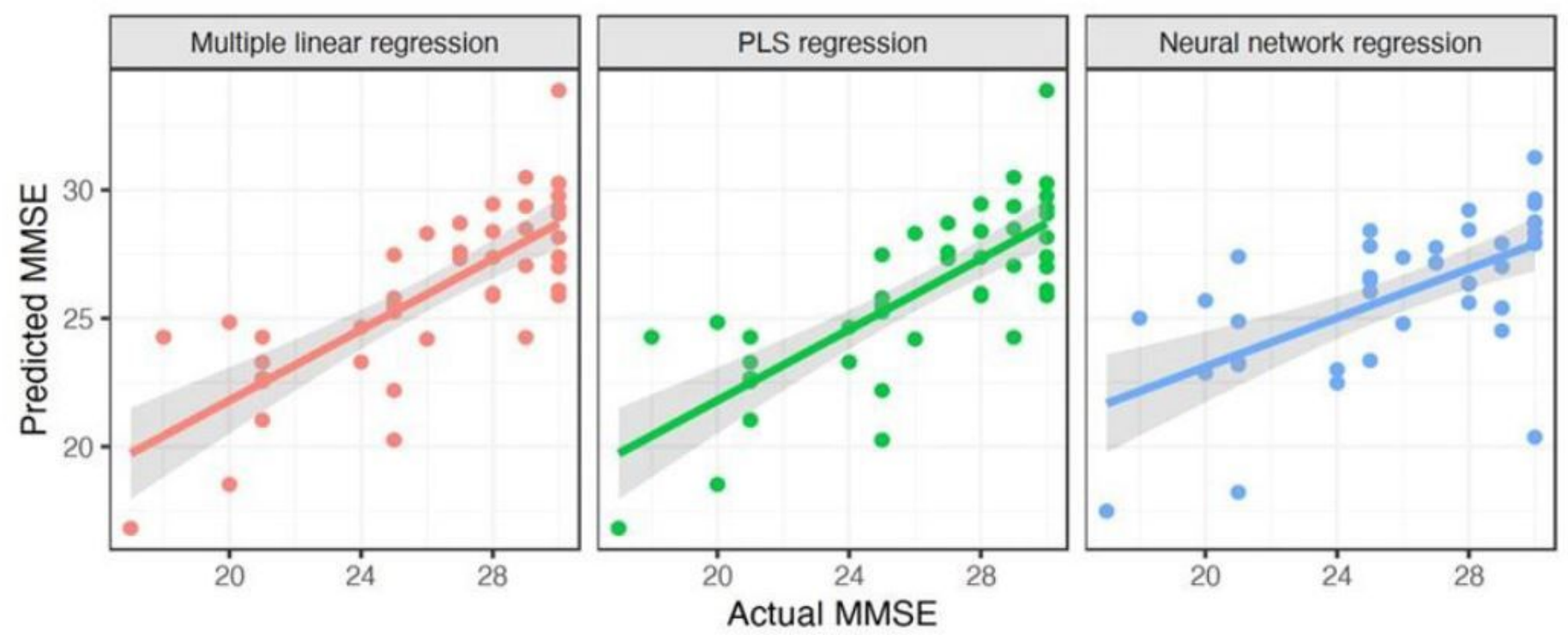

\section{Figure 4}

Scatter plot of predicted MMSE vs. actual MMSE of three prediction models (multiple linear regression, partial least squares (PLS) regression, and neural network regression models). The solid lines represent simple linear regression lines between actual and predicted MMSEs. The shaded area indicates the $95 \%$ confidence interval of each regression line. The prediction performance of each model was calculated by the leave-one-subject-out cross-validation. 